EDITORIAL

\title{
INFANCIA EN MOVIMIENTO
}

\section{CHILDHOOD IN MOVEMENT}

“El cuerpo humano está diseñado para estar en movimiento. Como profesionales de la salud debemos promover un estilo de vida más activo en nuestros pacientes", menciona el Comité de Graduados de la Sociedad Argentina de Diabetes (SAD), en esta edición dedicada a la actividad física.

Si de promoción de salud se trata, somos los pediatras, al intervenir en etapas tempranas de la vida, quienes más debemos comprometernos.

¿Cuáles son los efectos metabólicos de la actividad física en etapas tempranas? Estudios en niños con diabetes mellitus tipo 1 (DM1) describen menores valores de HbA1c, colesterol e índice de masa corporal (IMC) ${ }^{1}$. Más aún, los niños sanos más activos presentan menores niveles de péptido $\mathrm{C}$ independientemente del IMC².

Aunque la actividad física moderada a vigorosa ha dominado históricamente el debate, en Pediatría surge un enfoque más amplio e integrado, focalizado en la "conducta de movimiento en $24 \mathrm{~h}$ ", entendida como la relación entre actividad física, tiempo sedentario y horas de sueño, dado que estas dos últimas representan el 95\% del día y pueden contrarrestar los beneficios de la primera. Se describen siete posibles combinaciones. Los niños que cumplen las tres recomendaciones: 8 a 11 h de sueño, no más de $2 \mathrm{~h}$ diarias de pantallas recreativas y 60 min diarios de una actividad moderada a vigorosa constituyen el grupo con mayor beneficio para la salud ${ }^{4}$. Además estos componentes interactúan entre sí. La actividad aeróbica mejora la calidad de sueño ${ }^{5}$, el sueño inadecuado disminuye la actividad física y se asocia con mayor tiempo de pantallas, y este último se vincula con mayor ingesta y menor regulación del apetito6.

Los efectos benéficos de esta "conducta de movimiento en $24 \mathrm{~h}$ " modifican el desarrollo de la salud infantil más allá de lo metabólico y provocan efectos positivos a nivel neurocognitivo; los niños pequeños con menor tiempo sedentario presentan mayor memoria de trabajo a los 14 años ${ }^{7}$.

Al entender entonces la función central del movimiento para el desarrollo integral humano, conocer sus determinantes adversos resulta fundamental para cumplir adecuadamente nuestro rol.

Estudios en países desarrollados describen la disponibilidad de espacios abiertos y de aulas para pre-escolares ${ }^{8}$, etnicidad y nivel socio-económico9. En nuestro país, pensar en "infancia en movimiento" probablemente signifique asumir el desafío de asegurar una escolaridad adecuada y espacios seguros con la equidad que la infancia se merece.

\section{BIBLIOGRAFÍA}

1. Quirk H, Blake $H$, Tennyson R, Randell TL, Glazebrook C. Diabetes Med 2014; 31: 1163-1173.

2. Huus K, Akerman L, Raustrop A, Ludvigson J. Plos One 2016. Doi: 101371/journal.pone.0156401.

3. Chaput JP, Saunders TJ, Carson V. Interaction between sleep, movement and other non-movement behaviours in the pathogenesis of childhood obesity. Obesity Reviews 2017; 18 (Suppl 1): 7-14.

4. Saunders TJ, Gray CE, Poitras VJ, et al. Combinations of physical activity, sedentary behaviour and sleep: relationships with health indicators in school-aged children and youth. Appl Physiol Nutr Metab 2016; 41(6): S283-S293.

5. Kjeldsen JS, Rosenkilde M, Nielsen SW, et al. Effect of different doses of exercise on sleep duration, sleep efficiency and sleep quality in sedentary, overweight men. Bioenergetics 2013; 2: 108.

6. Thivel D, Chaput JP. Are post-exercise appetite sensations and energy intake coupled in children and adolescents? Sports Med 2014; 44: 735-741.

7. López-Vicente M, García-Aymerich J,Torrent-Pallicer J, et al. Are early physical activity and sedentary behaviours related to working memory at 7 and 14 years of age? J Pediatr 2017; 188: 35-41.

8. Olesen LG, Kristensen PL, Korsholm L, Froberg K. Physical activity in children attending preschools. Pediatrics 2013; 132: e1310-e1318.

9. Bowser J, Martínez-Donate AP, Carrel A, Allen DB, Moberg $P$. Disparities in fitness and physical activity among children. WMJ 2016; 115 (5): 245-250.

Dra. Gabriela Krochik

Especialista en Pediatría y Nutrición Pediátrica Jefa de Clínica del Servicio de Nutrición del Hospital de Pediatría J. P. Garrahan 